\title{
ON HAZARD RATE ORDERING OF DEPENDENT VARIABLES
}

\author{
EMAD-ELDIN A. A. ALY, ${ }^{*}$ University of Alberta \\ SUBHASH C. KOCHAR, ${ }^{* *}$ Indian Statistical Institute, New Delhi
}

\begin{abstract}
Shanthikumar and Yao (1991) introduced some new stochastic order relations to compare the components of a bivariate random vector $\left(X_{1}, X_{2}\right)$. As they point out in their paper, even if $X_{1} \geqq X_{2}$ according to their hazard rate (or likelihood ratio) ordering, the marginal distributions may not be ordered accordingly. We introduce some new concepts where the marginal distributions preserve the corresponding stochastic orders. Also a relation between the bivariate scale model and the introduced bivariate hazard rate ordering is established.
\end{abstract}

CONDITIONAL HAZARD RATE; BIVARIATE SCALE MODEL; STOCHASTIC ORDERING; CAUSE SPECIFIC HAZARD RATE; BIVARIATE INCREASING HAZARD RATE DISTRIBUTION

AMS 1991 SUBJECT CLASSIFICATION: PRIMARY $90 \mathrm{~B} 35$

\section{Introduction}

Let $X_{1}$ and $X_{2}$ be two independent random variables with survival functions $\bar{F}_{1}$ and $\bar{F}_{2}$, respectively. $X_{1}$ is said to be greater than $X_{2}$ according to the hazard rate ordering $\left(X_{1} \geqq_{\mathrm{hr}} X_{2}\right)$ if $\bar{F}_{1}(x) / \bar{F}_{2}(x)$ is non-decreasing in $x$. Keilson and Sumita (1982) call this ordering positive uniform stochastic ordering. If $X_{i}$ has a density function $f_{i}$ and failure rate function $r_{i}(\cdot)=f_{i}(\cdot) / \bar{F}_{i}(\cdot), i=1,2$, then $X_{1} \geqq_{\mathrm{hr}} X_{2} \Leftrightarrow r_{1}(\cdot) \leqq r_{2}(\cdot)$.

The likelihood ratio ordering, denoted by $\geqq_{\mathrm{Ir}}$, is defined as follows. $X_{1} \geqq_{1 \mathrm{r}} X_{2}$ if $f_{1}(x) / f_{2}(x)$ is non-decreasing in $x$. As usual $X_{1}$ is said to be stochastically larger than $X_{2}\left(X_{1} \geqq_{s t} X_{2}\right)$ if $\bar{F}_{1}(x) \geqq \bar{F}_{2}(x)$ for all $x$. As shown in Ross (1983), the hazard rate ordering is weaker than the likelihood ratio ordering but stronger than the stochastic ordering.

Following Shantikumar and Yao (1991) we define

$$
\mathscr{G}_{\mathrm{hr}}=\{g(x, y): \Delta(g(x, y)=g(x, y)-g(y, x) \text { is increasing in } x \text { for all } x \geqq y\} .
$$

In the case when $X_{1}$ and $X_{2}$ are independent Shanthikumar and Yao (1991) show that

$$
X_{1} \geqq X_{\mathrm{hr}} \Leftrightarrow \mathrm{E}\left\{g\left(X_{1}, X_{2}\right)\right\} \geqq \mathrm{E}\left\{g\left(X_{2}, X_{1}\right)\right\} \text { for all } g \in \mathscr{G}_{\mathrm{hr}} \text {. }
$$

Received 3 June 1992; revision received 28 September 1992.

* Postal address: Department of Statistics and Applied Probability, The University of Alberta, Edmonton, Alberta, Canada T6G 2G1.

Research supported by an NSERC Canada grant at the University of Alberta.

** Postal address: Indian Statistical Institute, 7, SJS Sansanwal Marg, New Delhi-110016, India.

Part of this research was done while visiting the University of Alberta supported by the NSERC Canada grant of the first author. 
Motivated by (1.2), they proposed an extension of the hazard rate ordering to the bivariate case, where $X_{1}$ and $X_{2}$ are jointly distributed with joint survival function $\bar{F}(x, y)=\boldsymbol{P}(X \geqq$ $x, Y \geqq y)$. Their definition is given below.

Definition 1.1. $X_{1}$ is greater than $X_{2}$ according to the joint hazard rate ordering $\left(X_{1} \geqq_{\mathrm{hr}: j} X_{2}\right)$ if and only if $\boldsymbol{E}\left\{g\left(X_{1}, X_{2}\right)\right\} \geqq E\left\{g\left(X_{2}, X_{1}\right)\right\}$ for all $g \in \mathscr{G}_{\mathrm{hr}}$.

From the proof of Theorem 3.17 of Shantihikumar and Yao (1991) it follows that $X_{1} \geqq_{\mathrm{hr}: \mathrm{j}} X_{2}$ if and only if

$$
\bar{F}(x, y)-\bar{F}(y, x) \text { is non-increasing in } y<x .
$$

In the case when $X_{1}$ and $X_{2}$ are independent we have $X_{1} \geqq_{\mathrm{hr}} X_{2} \Leftrightarrow X_{1} \geqq_{\mathrm{hr}: \mathrm{j}} X_{2}$.

Shanthikumar and Yao (1991) point out that, in general, there is no implication between $X_{1} \geqq_{\mathrm{hr}} X_{2}$ and $X_{1} \geqq_{\mathrm{hr}: \mathrm{j}} X_{2}$. This may result in situations where $X_{1} \geqq_{\mathrm{hr} ;} X_{2}$ but the hazard rate function of the marginal distribution of $X_{1}$ is not uniformly smaller than that of the marginal distribution of $X_{2}$.

In Section 2, we introduce and study a new concept of hazard rate ordering for the bivariate case. This new ordering is stronger than that of Definition 1.1, but it implies the hazard rate ordering of the marginal distributions. In Section 3 we study the relationship between the newly introduced ordering and the bivariate scale model. In the last section, some remarks are made on likelihood ratio ordering and the connection between hazard rate ordering and the comparison of cause specific hazard rates in the context of competing risks.

\section{A new concept of bivariate hazard rate ordering}

We use the notation $X_{1} \geqq_{\mathrm{HR}: \mathrm{B}} X_{2}$ to denote our new bivariate hazard rate ordering.

Definition 2.1.

$$
\begin{gathered}
X_{1} \underset{\mathrm{HR}: \mathrm{B}}{\geqq} X_{2} \Leftrightarrow \bar{F}(x, y) / \bar{F}(y, x) \text { is non-decreasing in } x \\
\quad \text { for all }(x, y) \text { such that } \bar{F}(y, x)>0 .
\end{gathered}
$$

The following theorem follows easily from (2.1).

Theorem 2.1

$$
\begin{gathered}
X_{1} \underset{\mathrm{HR}: \mathrm{B}}{\geqq} X_{2} \Leftrightarrow\left\{X_{1} \mid X_{2} \geqq y\right\} \underset{\mathrm{hr}}{\geqq}\left\{X_{2} \mid X_{1} \geqq y\right\} \text { for all } y, \\
X_{1} \underset{\mathrm{HR}: \mathrm{B}}{\geqq} X_{2} \Rightarrow X_{1} \geqq X_{\mathrm{hr}}, \\
X_{1} \underset{\mathrm{HR}: \mathrm{B}}{\geqq} X_{2} \Rightarrow \bar{F}(x, y) \geqq \bar{F}(y, x) \text { for } x \geqq y .
\end{gathered}
$$

As stated in the next theorem, the new bivariate hazard rate ordering is stronger than the hazard rate ordering of Shanthikumar and Yao (1991). This follows by noticing that the right-hand side of (2.1) implies (1.3).

Theorem 2.2. $X_{1} \geqq_{\mathrm{HR}: \mathrm{B}} X_{2} \Rightarrow X_{1} \geqq_{\mathrm{hr}: \mathrm{j}} X_{2}$.

The following result is a bivarite characterization of the bivariate hazard rate ordering. The proof of Theorem 2.3 follows from the bivariate characterization of $\left\{X_{1} \mid X_{2} \geqq y\right\} \geqq_{\mathrm{hr}}$ $\left\{X_{2} \mid X_{1} \geqq y\right\}$ for all $y>0$.

Theorem 2.3. $X_{1} \geqq_{\mathrm{HR}: \mathrm{B}} X_{2}$ if and only if for two independent random vectors $\left(\hat{X}_{1}, \hat{X}_{2}\right)$ and $\left(\hat{Y}_{1}, \hat{Y}_{2}\right)$ such that $\left(\hat{X}_{1}, \hat{X}_{2}\right) \stackrel{\text { fh }}{=}\left(\hat{Y}_{1}, \hat{Y}_{2}\right) \stackrel{\text { Df }}{=}\left(X_{1}, x_{2}\right)$, we have

$$
\boldsymbol{E}\left\{g\left(\hat{X}_{1}, \hat{Y}_{2}\right) I\left(\hat{Y}_{1} \wedge \hat{X}_{2} \geqq y\right\} \geqq \boldsymbol{E}\left\{g\left(\hat{Y}_{2}, \hat{X}_{1}\right) I\left(\hat{Y}_{1} \wedge \hat{X}_{2} \geqq y\right)\right\}\right.
$$


for all $y \geqq 0, g \in \mathscr{G}_{\mathrm{hr}}$, where $I(A)$ is the indicator function of the event $A, a \wedge b=\min (a, b)$ and $\mathscr{G}_{\mathrm{hr}}$ is as in (1.1).

An alternative characterization could be obtained using the results of Righter and Shanthikumar (1992).

Remarks 2.1. (a) It can be shown that $X_{1} \geqq_{\mathrm{hr}: \mathrm{j}} X_{2} \Rightarrow \bar{F}(x, y) \geqq \bar{F}(y, x)$ for $x \geqq y$.

(b) Suppose $\left(X_{1}, X_{2}\right)$ has the bivariate exponential distribution of Marshall and Olkin (cf. Barlow and Proschan (1981)) with $\lambda_{1} \leqq \lambda_{2}$. It can be shown that $X_{1} \geqq_{\mathrm{HR}: \mathrm{B}} X_{2}$.

(c) Suppose $\left(X_{1}, X_{2}\right)$ has absolutely continuous bivariate exponential distribution of Block and Basu (1974) with parameters $\lambda_{1}, \lambda_{2}$ and $\lambda_{12}$ such that $\lambda_{1} \leqq \lambda_{2}$. It can be shown that $X_{1} \geqq_{\mathrm{HR}: \mathrm{B}} X_{2}$.

\section{The bivariate scale model and the HR:B ordering}

In the case of independent random variables, Kochar (1979) proved the following result.

Let $F_{1}$ be IFR and let $F_{2}(x)=F_{1}(\sigma x)$. Then for $\sigma \geqq 1, X_{1} \geqq_{\mathrm{hr}} X_{2}$.

In this section we show that a similar result can be obtained in the case of jointly distributed random variables. First we discuss the bivariate scale model.

Let $H(x, y)$ be a bivariate distribution function satisfying

$$
H(x, y)=H(y, x) \text { for all }(x, y) .
$$

A random vector $\left(X_{2}^{\prime}, X_{2}^{\prime}\right)$ with distribution function $H(x, y)$ satisfying (3.1) is said to have a bivariate symmetric distribution. In this case $X_{1}^{\prime}$ and $X_{2}^{\prime}$ are also said to be exchangeable. Let $\sigma \geqq 1$ be a given constant and define a new distribution function $F(\cdot, \cdot)$ by

$$
F(x, y)=H(x, \sigma y) \text {. }
$$

The random vector $\left(X_{1}, X_{2}\right) \stackrel{\mathscr{g}}{=}\left(X_{1}^{\prime}, X_{2}^{\prime} / \sigma\right)$ with distribution function $F(\cdot, \cdot)$ satisfying (3.2) is said to follow a bivariate scale model. In this case $\left(X_{1}, \sigma X_{2}\right)$ is bivariate symmetric (independent of $\sigma$ ). The marginal distribution functions of $X_{1}$ and $X_{2}$ satisfy $F_{3}(t)=F_{1}(\sigma t)$ for all $t$.

Some common examples of bivariate scale model distributions are:

(i) The bivariate normal distribution with mean vector $(\mu, \mu)$ and variance covariance matrix

$$
\sum=\left[\sigma_{i j}\right], \quad \sigma_{11}=\sigma^{2} \geqq 1, \quad \sigma_{22}^{2}=1 \quad \text { and } \quad\left|\sigma_{12}\right|<\sigma .
$$

(ii) The absolutely continuous bivariate exponential distribution of Block and Basu (1974).

The following concept of positive dependence between two random variables is due to Harris (1970) (see also Barlow and Proschan (1981)).

Definition 3.1. A random vector $\left(X_{1}, X_{2}\right)$ is said to be right corner set increasing (RCSI) if $P\left[X_{1}>x, X_{2}>y \mid X_{1}>x^{\prime}, X_{2}>y^{\prime}\right]$ is increasing in $x^{\prime}$ and $y^{\prime}$ for each fixed $x$ and $y$.

Shaked (1977) has shown that $\left(X_{1}, X_{2}\right)$ is RCSI if and only if

$$
\left\{X_{1} \mid X_{2} \geqq y\right\} \text { is increasing in } y \text { in the hazard rate ordering, }
$$

or equivalently,

$\left\{X_{2} \mid X_{1} \geqq x\right\}$ is increasing in $x$ in the hazard rate ordering.

Using this result, we now prove the main result of this section.

Theorem 3.1. Let $\left(Y_{1}, Y_{2}\right)$ be an exchangeable random vector such that $\left(Y_{1}, Y_{2}\right)$ is RCSI and $\left\{Y_{2} \mid Y_{1} \geqq y\right\}$ is IFR for every $y$. Define $\left(X_{1}, X_{2}\right)$ by $X_{1} \stackrel{\text { 要 }}{=} Y_{1}$ and $X_{2} \stackrel{\text { g }}{=} Y_{2} / \sigma$ for some $\sigma>1$. 
Then

$$
X_{1} \underset{H R: B}{\geqq} X_{2} \text {. }
$$

Proof. Note that for every $x$,

$$
\begin{aligned}
\left\{X_{1} \mid X_{2} \geqq x\right\} & \stackrel{\mathscr{g}}{=}\left\{Y_{1} \mid Y_{2} \geqq \sigma x\right\} \\
& \geqq\left\{Y_{1} \mid Y_{2} \geqq x\right\} \stackrel{\mathscr{h}}{=}\left\{Y_{2} \mid Y_{1} \geqq x\right\} \\
& \geqq\left\{Y_{2} / \sigma \mid Y_{1} \geqq x\right\} \stackrel{\mathscr{g}}{=}\left\{X_{2} \mid X_{1} \geqq x\right\},
\end{aligned}
$$

where the first inequality above follows by (3.3) and the second inequality above follows by assumption that $\left\{Y_{2} \mid Y_{1} \geqq y\right\}$ is IFR.

By Theorem 2.1(a) and (3.5) we get (3.4).

\section{Some remarks}

4.1. On likelihood ratio ordering. Shanthikumar and Yao (1991) have also extended the concept of likelihood ratio ordering of two independent random variable to the bivariate case. According to their definition

$$
X_{1} \geqq X_{2} \Leftrightarrow f(x, y)-f(y, x) \geqq 0 \quad \text { for } \quad x \geqq y .
$$

Note that $X_{1} \geqq_{1 \mathrm{r}: j} X_{2}$ does not necessarily imply that the marginal distribution of $X_{1}$ is greater (according to the likelihood ratio ordering) than that of $X_{2}$. An alternative definition for a bivariate likelihhod ratio ordering could be formulated as follows:

$$
X_{1} \underset{\mathrm{LR}: \mathrm{B}}{\geqq} X_{2} \Leftrightarrow\left\{X_{1} \mid X_{2} \geqq y\right\} \underset{\mathrm{Ir}}{\geqq}\left\{X_{2} \mid X_{1} \geqq y\right\} \text { for all } y .
$$

By taking the limit as $y$ goes to $-\infty$ in the right-hand side of (4.1) we obtain $X_{1} \geqq_{\text {LR:B }} X_{2} \Rightarrow X_{1} \geqq \operatorname{lr} X_{2}$.

Next, we give a characterization of the bivariate likelihood ratio ordering.

Theorem 4.1. $X \geqq_{\mathrm{LR}: \mathrm{B}} X_{2}$ if and only if for two independent random vectors $\left(\hat{X}_{1}, \hat{X}_{2}\right)$ and $\left(\hat{Y}_{1}, \hat{Y}_{2}\right)$ such that $\left(\hat{X}_{1}, \hat{X}_{2}\right) \stackrel{\mathscr{g}}{=}\left(\hat{Y}_{1}, \hat{Y}_{2}\right) \stackrel{\mathscr{D}}{=}\left(X_{1}, X_{2}\right)$, we have

$$
\boldsymbol{E}\left\{g\left(\hat{X}_{1}, \hat{Y}_{2}\right) I\left(\hat{Y}_{1} \wedge \hat{X}_{2} \geqq y\right)\right\} \geqq \boldsymbol{E}\left\{g\left(\hat{Y}_{2}, \hat{X}_{1}\right) I\left(\hat{Y}_{1} \wedge \hat{X}_{2} \geqq y\right)\right\}
$$

for all $y \geqq 0$ and $g \in \mathscr{G}_{\mathrm{r}}:=\{g(u, v): g(u, v) \geqq g(v, u)$ for all $u \geqq v\}$.

The proof of Theorem 4.1 is parallel to that of Theorem 2.3 and is obtained by adapting the proof of Theorem 2.3 of Shanthikumar and Yao (1991).

It can be easily proved that

$$
X_{1} \underset{\mathrm{LR}: \mathrm{B}}{\geqq} X_{2} \Rightarrow X_{1} \underset{\mathrm{HR}: \mathrm{B}}{\geqq} X_{2},
$$

but we cannot establish any relationship between the orderings $\geqq_{\mathrm{rr:j}}$ and $\geqq_{\mathrm{LR}: \mathrm{B}}$.

Since HR:B ordering implies hr:j ordering, it follows from Theorem 4.9 of Shanthikumar and Yao (1991) that

$$
X_{1} \underset{\text { HR:B }}{\geqq} X_{2} \Rightarrow X \underset{\text { st: }}{\geqq} X_{2} \Rightarrow X_{1} \underset{\text { st }}{\geqq} X_{2},
$$

where $X_{1} \geqq_{\text {st: } j} X_{2}$ is defined in Shanthikumar and Yao (1991). 
4.2. On competing risks. There is a close connection between the conditional harzard rates as defined in Section 2 and the cause specific hazard rates (see Kalbfleisch and Prentice (1980)) which are defined below.

Let $\left(X_{1}, X_{2}\right)$ have joint distribution function $F(x, y)$ and define $T=\min \left(X_{1}, X_{2}\right)$ and $\delta=2-I\left\{X_{1} \leqq X_{2}\right\}$. The cause specific hazard rate (CSHR) corresponding to the $i$ th cause (i.e. $X_{i}$ ) is defined as

$$
g_{i}(t)=\lim _{\Delta t \rightarrow 0} \frac{1}{\Delta t} \mathrm{P}\{t \leqq T<t+\Delta t, \delta=i \mid T \leqq t\}, \quad i=1,2 .
$$

Note that

$$
\begin{aligned}
g_{1}(t) & =\int_{t}^{\infty} f(t, y) d t / \bar{F}(t, t) \\
& =-\left.\frac{\partial}{\partial x} \ln \bar{F}(x, t)\right|_{x=t}=r_{1}\left(t \mid X_{2} \geqq t\right)
\end{aligned}
$$

and $g_{2}(t)=r_{2}\left(t \mid X_{1} \geqq t\right)$.

Theorem 4.2

$$
\bar{F}(x, y) \geqq \bar{F}(y, x) \text { for all } x \geqq y \Rightarrow g_{1}(\cdot) \leqq g_{2}(\cdot)
$$

Proof. Let $\psi(x, t)=\ln (\bar{F}(x, t) / \bar{F}(t, x))$ and note that $\psi(t, t)=0$. Observe that by Theorem 4.2

$$
\psi(t+s, t)-\psi(t, t) \geqq 0 \quad \text { for all } t \text { and } s \geqq 0 .
$$

Hence

$$
\lim _{s \rightarrow 0} \frac{1}{S}\{\psi(t+s, t)-\psi(t, t\} \geqq 0 \quad \text { for all } \quad t \geqq 0
$$

Consequently,

or

$$
\left.\frac{\partial}{\partial y} \psi(y, t)\right|_{y=t} \geqq 0 \quad \text { for all } \quad t \geqq 0
$$

$$
g_{1}(t) \leqq g_{2}(t) \text { for all } t \geqq 0 .
$$

It follows from Theorem 2.1(c) and the above theorem that, in particular, $X_{1} \geqq_{\mathrm{HR}: \mathrm{B}} X_{2} \Rightarrow$ $g_{1}(\cdot) \leqq g_{2}(\cdot)$

\section{Acknowledgment}

We wish to thank the referee for his comments and suggestions which improved the presentation.

\section{References}

Barlow, R. E. and Proschan, F. (1981) Statistical Theory of Reliability and Life Testing. To Begin With, Silver Spring, Maryland.

Block, H. and Basu, A. P. (1974) A continuous bivariate exponential extension. J. Amer. Statist. Assoc. 69, 1031-1037.

Harris, R. (1970) A multivariate definition for hazard rate distributions. Ann. Math. Statist. 41, 413-417.

Kalbfleisch, J. D. And Prentice, R. L. (1980) The Statistical Analysis of Failure Time Data. Wiley, New York. 
KeILSON, J. AND Sumita, U. (1982) Uniform stochastic ordering and related inequalities. Canad. J. Statist. 10, 181-198.

KochaR, S. C. (1979) Distribution-free comparison of two distributions with reference to their hazard rates. Biometrika $66,437-441$.

Righter, R. AND Shanthikumar, J. G. (1992) Extension of the bivariate characterization for stochastic orders. Adv. Appl. Prob. 24, 506-508.

Ross, S. (1983) Stochastic Processes. Wiley, New York.

SHAKED, M. (1977) A family of concepts of dependence for bivariat distributions. J. Amer. Statist. Assoc. 72, 642-650.

Shanthikumar, J. G. AND YAO, D. D. (1991) Bivariate characterization of some stochastic order relations. Adv. Appl. Prob. 23, 642-659. 